\title{
Tracheal bronchus presenting with recurrent haemoptysis in an adult female
}

The authors declare no financial disclosure

\begin{abstract}
Abnormal bronchus arising directly from the trachea or the main bronchus is a rare developmental anomaly of the tracheobronchial tree. In general, tracheal bronchus has been reported in less than $1 \%$ of adult patients undergoing bronchoscopy with a male predominance. Tracheal bronchus is classified as — 'displaced' and 'supernumerary', the former being the most common type reported. Most patients are asymptomatic; however, cases presenting with cough, haemoptysis or recurrent lung infections are not uncommon. The diagnosis is usually made through computed tomography or bronchoscopy. Awareness of this anomaly may help in timely identification or prevention of perioperative complications during general anaesthesia or resectional thoracic surgery. Herein we report a case of right-sided displaced tracheal bronchus in an adult female presenting with recurrent haemoptysis.
\end{abstract}

Key words: congenital anomaly, tracheal bronchus, haemoptysis, flexible bronchoscopy

Adv. Respir. Med. 2017; 85: 155-157

\section{Introduction}

Abnormal bronchus arising directly from the trachea or the main bronchus is a much rarer anomaly compared to diverse anatomic variations seen in the branching pattern of lobar or segmental bronchi in routine clinical practice. Tracheal bronchus and accessory cardiac bronchus are the two most common developmental anomalies of the tracheobronchial tree. Tracheal bronchus was first reported by Sandifort in the year 1785 as a right upper lobe bronchus originating from the trachea. In recent time, the term tracheal bronchus is used to describe any bronchus that arises directly from the trachea or the main bronchus and provides ventilation to the upper lobes $[1,2]$.

The prevalence of tracheal bronchus has been reported as $0.1-2 \%$ on the right and $0.3-1 \%$ on the left side [2]. The anomaly was found in 8 out of $12,648(0.06 \%)$ adult patients undergoing flexible bronchoscopy in the Turkish population [3]. It is mostly located on the right side and is three times more common in males than females [2-4]. Tracheal bronchus is often detected accidentally during computed tomography (CT) in asymptomatic individuals. Suzuki et al. reported tracheal bronchus in 30 cases out of 9781 (0.31\%) CT images with a male-female ratio of 2:1 [5]. Symptomatic patients usually present with cough, dyspnoea and haemoptysis [3]. However, cases with recurrent lung infections, persistent cough, stridor, and acute respiratory distress have been reported. Bronchiectasis, atelectasis, focal emphysema, cystic malformations may coexist with tracheal bronchus [2]. Interestingly, one case of bilateral true tracheal bronchus in an adult presenting with haemoptysis has been reported earlier [6]. We report here a case of a right-sided tracheal bronchus in an adult female presenting with recurrent haemoptysis.

\section{Case report}

A 35-year-old female patient presented with four episodes of scanty haemoptysis within 20 days.

Address for correspondence: Manoj Kumar Panigrahi, Pulmonary Medicine All India Institute of Medical Sciences, Bhubaneswar, India, e-mail: panigrahimanoj75@gmail.com DOI: 10.5603/ARM.2017.0026

Received: 25.10.2016

Copyright (C) 2017 PTChP

ISSN 2451-4934 
She reported a similar episode of non-massive haemoptysis the previous year. She had not suffered from pulmonary tuberculosis or any allergic disorders in the past. She never smoked nor had any substance abuse. She was married and had two healthy children. Physical examination and lung auscultation were unremarkable. Haemogram showed haemoglobin of $10 \mathrm{~g} / \mathrm{dl}$, white cell count $8500 / \mathrm{mm}^{3}$ with differentials of neutrophils $63 \%$, lymphocytes $35 \%$, eosinophils $2 \%$. Her bleeding time was 1 min 15 seconds, clotting time 3 minutes, and prothrombin time-international

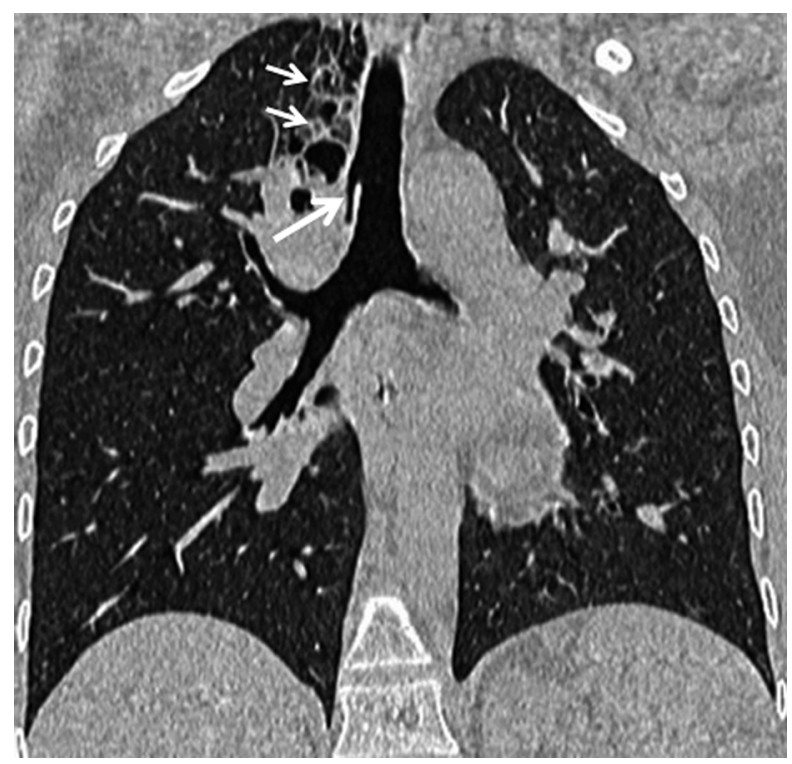

Figure 1. Coronal section computed tomography image showing the right tracheal bronchus (long arrow) arising from the lateral wall of the trachea about $3.5 \mathrm{~cm}$ proximal to the carina with areas of atelectasis and bronchiectasis (short arrows) in the apical segment of the upper lobe. Rest of the lung parenchyma bilaterally appears normal normalised ratio of 1 . Blood glucose, renal and liver function tests were normal. Chest radiograph was apparently within the norm. High resolution CT scan showed an accessory bronchus arising from the trachea $3.57 \mathrm{~cm}$ proximal to the carina (Fig. 1, long arrow), directed towards the apical segment of the right upper lobe that showed bronchiectatic changes (Fig. 1, short arrows). Flexible bronchoscopy demonstrated the origin of a tracheal bronchus on the right lateral wall of the trachea proximal to the carina (Fig. 2A, arrow) and the right upper lobe bronchus divided into two segmental branches (Fig. 2B) instead of usual triple divisions. Bronchoalveolar lavage collected from the tracheal bronchus orifice was non-contributory factor in bacterial and mycobacterial infections. The patient was treated with a course of oral amoxicillin-clavulanic acid preparation, ethamsylate and codeine syrup as cough suppressant. Screening abdominal sonography and echocardiogram excluded any other associated congenital anomalies. The patient improved symptomatically and further haemoptysis was not reported until the last contact a month ago. Future requirement of surgical lung resection was discussed with the patient in case of recurrent lung infections or massive haemoptysis.

\section{Discussion}

Tracheal bronchus can be of 'displaced' or 'supernumerary' type. When the presence of a tracheal bronchus is associated with an absence of a segmental branch in the upper lobe, it is known as 'displaced' type. In other words, a displaced type of tracheal bronchus represents a normal segmental bronchial division of the upper lobe
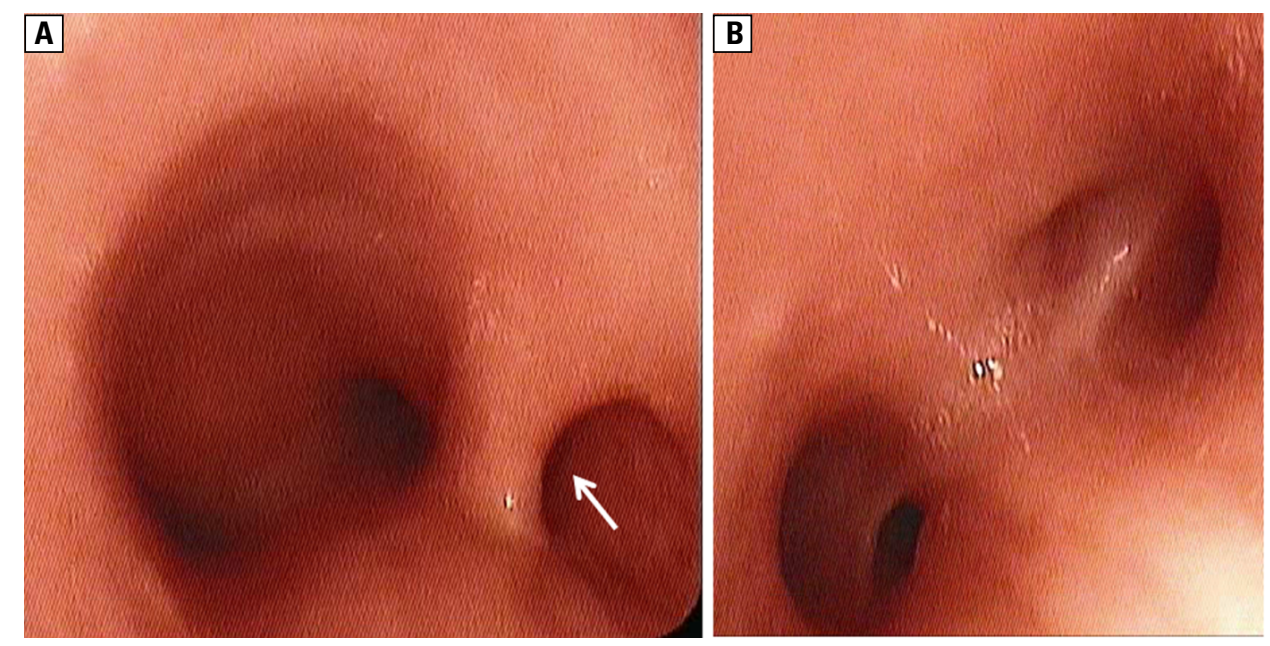

Figure 2. (A) Flexible bronchoscopy image near lower one third of the trachea showing the orifice of tracheal bronchus (arrow) arising from the right posterolateral wall of the trachea proximal to the carina (B) Right upper lobe bronchus divides into two segmental branches instead of three 
of aberrant origin. The 'supernumerary' type is a true accessory bronchus as it is associated with normal branching pattern of the upper lobe bronchus [2]. The displaced type occurs more frequently than the supernumerary one [1-5]. Usually, displaced tracheal bronchus ventilates the apical segment of the upper lobe on the right side and apicoposterior segment of the upper lobe on the left side. When the right upper lobe bronchus itself arises from the trachea and divides into segmental and subsegmental branches, it is called 'pig bronchus' and is encountered very rarely, namely in $0.2 \%$ of cases [2]. Normally, the right upper lobe bronchus originates above the right pulmonary artery, hence it is called eparterial, and the left upper lobe bronchus is called hyparterial as it originates below the left pulmonary artery. Tracheal bronchus proximal to the origin of the upper lobe bronchus is called preparterial on the right side and eparterial or prehyparterial on the left side. Anomalous bronchus arising below the origin of the upper lobe bronchus is called posteparterial on the right side and posthyparterial on the left side [2]. The tracheal bronchus in our case is a displaced preparterial type - the variant most commonly reported in many series [1-5].

The tracheal bronchus arises from the trachea usually within $2-6 \mathrm{~cm}$ proximal to the carina $[1,2]$. In our case the accessory bronchus originated from the trachea $3.57 \mathrm{~cm}$ proximal to the carina. Several other anomalies such as right main bronchus stenosis, infantile lobar emphysema, tracheoesophageal fistula, sling left pulmonary artery, congenital diaphragmatic hernia, trisomy 21 , and upper rib anomalies have been described in association with tracheal bronchus [3]. However, no such anomaly was found in our case.

Tracheal bronchus has significant implications during elective or emergency anaesthesia. Unrecognised intubation of a tracheal bronchus can cut off the ventilation to the rest of the lung resulting in low tidal volume, hypoxaemia and respiratory acidosis; whereas bypassing the accessory bronchus during intubation may result in postoperative collapse of the lung ventilated by the tracheal bronchus [7, 8]. Occasionally, cases of lung cancer, carcinoid tumour and squamous cell carcinoma arising from the tracheal bronchus have been reported [9-11]. Treatment depends on the severity of symptoms. Most patients can be managed conservatively and surgical excision of the involved segment is required only in the presence of recurrent pneumonia, atelectasis or air trapping of the upper lobe [3].

\section{Conclusions}

Tracheal bronchus is a rare developmental anomaly of the bronchial tree. Most patients with tracheal bronchus are asymptomatic; however, they may present with cough and haemoptysis as in our case. Bronchiectasis of the apical lung segments ventilated by abnormal bronchus may lead to recurrent haemoptysis. CT scan and bronchoscopy are essential for classifying the type of tracheal bronchus. Awareness of the disorder may help in prevention or early diagnosis and management of unforeseen perioperative complications.

\section{Conflict of interest}

The authors declare no conflict of interest.

\section{References:}

1. Desir A, Ghaye B. Congenital abnormalities of intrathoracic airways. Radiol Clin North Am. 2009; 47(2): 203-225, doi: 10.1016/j.rcl.2008.11.009, indexed in Pubmed: 19249452.

2. Ghaye B, Szapiro D, Fanchamps JM, et al. Congenital bronchial abnormalities revisited. Radiographics. 2001; 21(1): 105-119, doi: 10.1148/radiographics.21.1.g01ja06105, indexed in Pubmed: 11158647.

3. Findik S. Tracheal bronchus in the adult population. J Bronchology Interv Pulmonol. 2011; 18(2): 149-153, doi: 10.1097 LBR.0b013e318216e30e, indexed in Pubmed: 23169084.

4. Ulusoy M, Kivrak A, Uysal I, et al. Developmental Anomalies of Bronchial Tree: A Multidetector Computerized Tomography Study. International Journal of Morphology. 2013; 31(3): 10491055, doi: 10.4067/s0717-95022013000300044.

5. Suzuki M, Matsui O, Kawashima H, et al. Radioanatomical study of a true tracheal bronchus using multidetector computed tomography. Jpn J Radiol. 2010; 28(3): 188-192, doi: $10.1007 /$ s11604-009-0405-5, indexed in Pubmed: 20437128.

6. Kumagae Y, Jinguji M, Tanaka D, et al. An adult case of bilateral true tracheal bronchi associated with hemoptysis. J Thorac Imaging. 2006; 21(4): 293-295, doi: 10.1097/01. rti.0000213557.46505.9b, indexed in Pubmed: 17110854 .

7. Choi YS, Kwak YL, Choi HG, et al. Anesthetic experience of an adult patient with an unrecognized tracheal bronchus -A case report-. Korean J Anesthesiol. 2010; 59 Suppl: S13S16, doi: 10.4097/kjae.2010.59.S.S13, indexed in Pubmed: 21286422.

8. O'Sullivan BP, Frassica JJ, Rayder SM. Tracheal bronchus: a cause of prolonged atelectasis in intubated children. Chest. 1998; 113(2): 537-540, indexed in Pubmed: 9498980.

9. Kuo CW, Lee YC, Perng RP. Tracheal bronchus associated with lung cancer: a case report. Chest. 1999; 116(4): 1125-1127, indexed in Pubmed: 10531184.

10. Patrinou V, Kourea H, Dougenis D. Bronchial carcinoid of an accessory tracheal bronchus. Ann Thorac Surg. 2001; 71(3): 1034-1035, indexed in Pubmed: 11269426.

11. Nicolaou N, Du Plessis A. Squamous carcinoma arising from a true tracheal bronchus: Management and case report. Int J Surg Case Rep. 2015; 6C: 256-258, doi: 10.1016/j. ijscr.2014.12.005, indexed in Pubmed: 25549955. 